there is no projection on $(m)$ to $(C)$, it may be shown that at least either there is no projection on $(m)$ to $Y$, or else there is no projection on $(m)$ to the complementary subspace of $Y$ in $(C)$. (An illustration of the case where there is no projection on $(m)$ to the complementary subspace in $(C)$ is provided by the case of a finite dimensional $Y \subset(C)$.)

In a paper in preparation on the extension of linear transformations, the writer intends to discuss the questions indicated above, and related questions.

Oregon State College

\title{
SEQUENCES OF STIELTJES INTEGRALS ${ }^{1}$
}

\section{H. M. SCHWARTZ}

Statement of results. Sequences of Riemann-Stieltjes integrals ${ }^{2}$ have as yet been little studied, only the following fundamental results being known.

Theorem A (Helly [2]). Let $g_{n}(x)(n=1,2, \cdots)$ be an infinite sequence of real functions defined in the finite closed interval $I=(a, b)$ which satisfy the following two conditions:

$$
\begin{aligned}
& \text { Total variation of } g_{n} \text { in } I \equiv V_{I}\left(g_{n}\right) \leqq M, \quad M \text { a fixed constant, } \\
& g_{n} \rightarrow g \text { on } I \text {, } \\
& n \rightarrow \infty ;
\end{aligned}
$$

then for any function $f(x)$ continuous in $I$, we have

$$
\int f d g_{n} \rightarrow \int f d g .
$$

THEOREM B (Shohat [3]). Let $\left\{g_{n}\right\}$ be a sequence of functions monotonic and uniformly bounded in $I$ and such that

(4) $g_{n} \rightarrow g$ on $E, E$ a set dense on $I$ and including the end points $a, b$ of $I$,

where $g$ is a monotonic function (all the functions $g_{n}, g$ monotonic in the same sense); then we have (3) for any function $f(x)$ for which

\footnotetext{
1 Presented to the Society, January 1, 1941.

2 A discussion of such integrals with references is to be found in [1]. (Numbers in brackets refer to the bibliography.)

${ }^{3}$ When the limits of integration are omitted, it is to be understood that they are the end points $a, b$ of $I$.
} 


$$
\begin{aligned}
& \int f d g_{n} \text { exist, } \quad n=1,2, \cdots, \\
& \int f d g \text { exists. }
\end{aligned}
$$

Theorem A was rediscovered by Bray [4] $]^{4}$ and a condition weaker than (4), which is both necessary and sufficient, was found by Hildebrandt $[5] .{ }^{5}$

The present paper is devoted to a preliminary study of conditions governing convergence of the sequences in question when neither continuity of $f$ nor monotonicity of $g_{n}$ is assumed. The goal, not attained here, is to find necessary and sufficient conditions to be imposed on the sequence $\left\{g_{n}\right\}$, where the functions $g_{n}$ belong to the class $V$ of functions of bounded variation in $I$, in order to insure the validity of (3) for every bounded $f$ for which (5), or (5) and (6) hold true. Two necessary conditions can be given at once. Condition (1) is necessary since it is already necessary if we require that $f$ belong only to the class of functions continuous in $I[5]$. It is interesting to note, however, that condition (1) is not necessary if we require that $f$ belong only to $V$, as is seen by the following theorem:

THEOREM 1. If we have (2), then in order that we have (3) for every function $f$ of $V$ for which (5) and (6) are true, it is sufficient that the sequence $\left\{g_{n}\right\}$ be uniformly bounded.

The other necessary condition is obtained by taking for $f$ functions which are constant everywhere in $I$ except for a single point. It is easily found that the set $C$ of points of convergence of $g_{n}$ to $g$ must include all the common continuity points of $g_{n}, g$ as well as the end points $a, b$ of $I$. Without going into the question of necessity ${ }^{6}$ we shall assume that $C$ contains all the continuity points of $g$ (whether they are also continuity points of all $g_{n}$ or not), that is, that

$$
g_{n} \rightarrow g \text { on } C, \quad g \text { in } V,
$$

$C$ consisting of the continuity points of $g$ and of $a$ and $b$.

${ }^{4}$ In Bray's formulation of the theorem condition (2) is replaced by (4); but it is then necessary to specify that $g$ is a function of bounded variation; and by the lemma given below, it is seen that the two formulations are completely equivalent.

s The following additional reference was brought to the author's attention by the referee: Hildebrandt, Stieltjes integration of the Riemann type, American Mathematical Monthly, vol. 45 (1938), pp. 265-278, Theorems $2.41 ; 5.41$.

${ }^{6} \mathrm{It}$ is to be noted that in Theorem B as well as in its generalization, Theorem 3, conditions (4) and (7) are equivalent, as can be readily proved. 
The question arises whether condition (7) represents in the problem under consideration an essential weakening of condition (2). That this is not the case is shown by the following lemma.

Lemma. Under the conditions (1), (5), and (6), if (3) is implied by (2), it will also be implied by (7). If we are considering only a certain $f$ satisfying our conditions, then $C$ need consist of only those continuity points of $g$ which are also discontinuity points of $f$ (in addition to being dense on $I$ and containing the points $a$ and $b$ ).

It is thus no loss of generality to assume condition (2) rather than (7) in the following theorems. When this is the only condition imposed on the sequence $\left\{g_{n}\right\}$, only the following result (besides that given in Theorem 1) has so far been obtained.

Theorem 2. If $f$ has at most singularities of the first kind, then (1), (2), (5), and (6) imply (3).

A direct generalization of Theorem B is given in the following theorem.

Theorem 3. If in addition to (2) we assume that

$$
V_{I}\left(g_{n}\right) \rightarrow V_{I}(g),
$$

$g_{n}, g$ in $V$,

then (5) and (6) imply (3). (Condition (1) is of course implied by (8).)

The next theorem gives a result of wider scope.

THEOREM 4. To insure the validity of (3) it is sufficient to assume in addition to (1), (2), and (5) that ${ }^{7}$

(9) for every limit function $v(x)$ of the sequence $\left\{V_{a}^{x}\left(g_{n}\right)\right\} \int$ fdv exists.

In some applications the interval of integration is infinite and it is therefore of interest to consider that case. This is treated here only briefly, the following results being immediate. ${ }^{8}$

Theorem 5. For $I=(a, b)=(a, \infty)$, if we assume (2), (5), and

$$
\int_{a}^{c} f d g_{n} \rightarrow \int_{a}^{c} f d g \text { for every finite } c>a ; \quad \int_{a}^{c} f d g \text { exists, }
$$

${ }^{7}$ The existence of at least one limit function of the sequence given in (9) is insured by Helly's theorem of choice [2]. Note also that (6) is implied by (9), as can be seen, for example, by using the integrability condition on p. 542 [1] along with Theorem 2 in [7].

${ }^{8}$ In order to simplify the formulas, we take only one of the limits as infinite. 
then a necessary and sufficient condition for the validity of (6) and (3) is that we have either one of the following two conditions ( $e$ being an arbitrarily assigned positive number):

$$
\begin{array}{r}
\left|\int_{c}^{\infty} f d g_{n}\right|<e, \quad c \geqq c_{e}, n \geqq N(e, c), \\
\left|\int_{a}^{c} f d\left(g_{n}-g\right)\right|<e, \quad n \geqq n_{e}, c \geqq C(e, n) .
\end{array}
$$

Note 1. Condition (10) of the theorem can of course be replaced by the appropriate conditions in the preceding theorems which insure the validity of $(10)$; thus if $f$ is continuous in $I$, we can replace (10) by the requirement:

$$
V_{a}^{c}\left(g_{n}\right) \leqq M(c) .
$$

Note 2 . If $f$ is bounded in $I$, then (11a) can be replaced by the relation $V_{c}^{\infty}\left(g_{n}\right)<e, c \geqq c_{e}, n \geqq N(e, c)$.

Note 3. If $f$ is bounded in $I$ and $g$ is of bounded variation for sufficiently large $x$, then we need assume only the validity of a relation like (4), and (11) can be replaced by the following corresponding relations:

$$
\begin{array}{r}
\left|\int_{a_{k}}^{\infty} f d g_{n}\right|<e, \quad k \geqq k_{e}, n \geqq N(e, k), \\
\left|\int_{a}^{a_{k}} f d\left(g_{n}-g\right)\right|<e, \quad n \geqq n_{e}, k \geqq K(e, n),
\end{array}
$$

where $\left\{a_{k}\right\}$ is some sequence in $E$ converging to $\infty$.

Note 4. If we have (12) and if

$$
\lim _{c \rightarrow \infty} \bar{B}\{|f(x)|\} \cdot M(c)=0,
$$

then we certainly have (11a). (The above relation will hold for instance when $\lim _{x \rightarrow \infty} f(x)=0$ and $M(c)$ is independent of $c$.)

By the use of Theorem 5 we can give a direct proof of the existence of a solution of the moments-problem of Stieltjes-Hamburger. ${ }^{9}$ This proof as well as other applications of the above theorems will be given elsewhere.

Proof of the lemma. The proof will be based on the following auxiliary lemma.

${ }^{9}$ The proof given in the literature is indirect, involving complex function theory. A discussion of this problem and references are to be found in [6]. 
LEMMA. Let $g$ and $h$ be two functions of $V$ which coincide in value on a set $D$, and let $f$ be a function bounded in $I$. Then the existence of the integral of $f$ with respect to one of these functions, say $g$, will imply its existence with respect to the other function $h$, and the equality of the values of the two integrals, provided $D$ is dense in I and includes the end points of $I$ as well as all those discontinuity points of $f$ at which h has an external saltus.

Proof. That $\int f d g=\int f d h$ when both integrals exist follows directly by the definition of Stieltjes integrals and the fact that $D$ is dense in $I$ and includes the end points of $I$. To prove that $\int f d h$ exists it is clearly sufficient to prove that $\int f d k$ exists, where $k=g-h$; and for that it is only necessary to show that the variation of $k$ over the set of discontinuities of $f$ is zero [1]. This can be readily shown when we note that by our assumption $k(x-0)=k(x+0)=0$, and that therefore, if we denote the discontinuity points of $k$ by $u_{i}(i=1,2, \cdots)$, the total variation of $k$ over the set obtained by excluding from $I$ arbitrarily small intervals about the points $u_{1}, \cdots, u_{m}$, can be made as small as we please provided $m$ is taken sufficiently great; and that further by our assumptions, $f$ is continuous at each $u_{i}$.

To prove the lemma, we note that by (1) the sequence $\left\{\int f d g_{n}\right\}$ $\equiv\left\{J_{n}\right\}$ is bounded and it therefore contains at least one convergent subsequence. Let $\left\{J_{n^{\prime}}\right\}$ be any such convergent subsequence and denote its limit by $J$; let $\left\{g_{n^{\prime \prime}}\right\}$ be a convergent subsequence of the function-sequence $\left\{g_{n^{\prime}}\right\}$ (existing by Helly's theorem of choice [2]), and denote its limit function by $g^{*}$. Now it can easily be proved that by the condition given in the lemma, no limit function of $\left\{g_{n}\right\}$ can have an external saltus at a discontinuity point of $f$. We can apply therefore the above auxiliary lemma and conclude that $\int f d g^{*}$ exists and equals $\int f d g$. Hence by our assumption, it follows that $\int f d g_{n^{\prime \prime}} \rightarrow \int f d g$, and consequently also $J=\int f d g$. But $\left\{J_{n^{\prime}}\right\}$ was an arbitrary convergent subsequence of $\left\{J_{n}\right\}$; hence $\left\{J_{n}\right\}$ has the unique limit $J$, that is, we have (3).

Proof of Theorem 1. It is clearly sufficient to consider $f$ monotonic. By the formula of integration by parts for Stieltjes integrals [1], it is seen that the theorem will follow if we show that

$$
\int g_{n} d f \rightarrow \int g d f
$$

To this end, we employ Lebesgue's theorem on the convergence of sequences of Lebesgue integrals with boundedly convergent integrands. 
Putting $A=f(a), B=f(b), G_{n}(y) \equiv g_{n}\left(f^{-1}(y)\right), G(y) \equiv g\left(f^{-1}(y)\right)$, we have [1]:

$$
\int g_{n} d f=L \int_{A}^{B} G_{n}(y) d y, \quad \int g d f=L \int_{A}^{B} G(y) d y ;
$$

and as by our assumptions, all the conditions of Lebesgue's theorem are satisfied, it follows that $\int G_{n}(y) d y \rightarrow \int G(y) d y$, and that we have $\left(^{*}\right)$.

Proof of Theorem 2. This theorem follows from the following three lemmas (Lemma 2 is more general than the present use of it requires).

LemMA 1. Theorem 2 is true if $f$ is in $V$.

Proof. Let $f=f_{c}-f_{d}$ be the decomposition of $f$ into its continuous part $f_{c}$ and its discontinuous part $f_{d}[1]$. By Theorem $\mathrm{A}, \int f_{c} d g_{n} \rightarrow \int f_{c} d g$, and it remains to be shown that we also have the relation $\int f_{d} d g_{n} \rightarrow \int f_{d} d g$, or the relation corresponding to $\left(^{*}\right)$ above. Now $\int g_{n} d f_{d}=\sum_{m} d_{m} g_{n}\left(u_{m}\right)$, where $d_{m}=f\left(u_{m}+0\right)-f\left(u_{m}-0\right)$, and as the series $\sum d_{m} g_{n}\left(u_{m}\right)$ converges uniformly with respect to $n$, being dominated by

$$
\bar{B}\left\{\left|g_{n}\right|\right\} \cdot \sum\left|d_{m}\right|
$$

which is finite by our assumptions, it follows that

$$
\lim _{n \rightarrow \infty} \int g_{n} d f_{d}=\sum_{m} d_{m} g\left(u_{m}\right)=\int g d f_{d}
$$

LEMMA 2. If the sequence of bounded functions $f_{m}$ converges uniformly in $I$ to the bounded function $f$, and if we have (5), (6), and (3) with $f$ replaced by $f_{m}(m=1,2, \cdots)$ then we have (5), (6), and (3), provided we assume (1) and the finiteness of $V_{I}(g)$.

Proof. We show first that the existence of $\int f_{m} d g$ and the uniform convergence of $f_{m}$ to $f$ in $I$ implies the relation

$$
\int f d g \text { exists, } \quad \int f_{m} d g \rightarrow \int f d g,
$$

$g$ being assumed to belong to $V$. Let

$$
s_{m k}=\sum_{i=1}^{r_{k}} f_{m}\left(u_{i}\right)\left[g\left(x_{i}\right)-g\left(x_{i-1}\right)\right]
$$

be the $k$ th Riemann sum of a sequence whose limit for $k \rightarrow \infty$ is $\int f_{m} d g$, and let $s_{k}$ represent the corresponding sum for $f$. As

$$
\left|s_{m k}-s_{k}\right| \leqq \underset{x \text { in } I}{\bar{B}}\left\{\left|f_{m}-f\right|\right\} \cdot V_{I}(g),
$$


we see that $s_{m k}$ approaches its limit $s_{k}$ uniformly with respect to $k$, and that therefore $\lim _{k \rightarrow \infty} s_{k}$ and $\lim _{m \rightarrow \infty} \int f_{m} d g$ exist and are equal. Relation $\left({ }^{*}\right)$ now follows from the fact that the first limit is independent of the choice of the division points $x_{i}$ and the between-points $u_{i}$ in the Riemann sums.

Now by (1),

$$
\left|\int f d g_{n}-\int f_{m} d g_{n}\right| \leqq \bar{B}_{x \text { in } I}\left\{\left|f-f_{m}\right|\right\} \cdot M,
$$

that is, $\int f_{m} d g_{n}$ converges to $\int f d g_{n}$ uniformly with respect to $n$. Hence

$$
\begin{aligned}
\lim _{n} \int f d g_{n} & =\lim _{n} \lim _{m} \int f_{m} d g_{n}=\lim _{m} \lim _{n} \int f_{m} d g_{n} \\
& =\lim _{m} \int f_{m} d g=\int f d g
\end{aligned}
$$

LEMMA 3. If $f$ is a function having at most singularities of the first kind in $I$, then there exists a sequence $\left\{f_{m}\right\}$ converging to $f$ uniformly in $I$, where each $f_{m}$ is in $V$ and is continuous at the continuity points of $f$.

Proof. We have to show that we can assign to every given positive and arbitrarily small number $e$, a function $f_{e}$, which is in $V$, is continuous at every continuity point of $f$, and approximates $f$ within $e$. By assumption, $f(x-0)$ and $f(x+0)$ exist, and we can therefore assign to every $x$ of the semi-open interval $(a, b],{ }^{10}$ a left-side interval $\left(x^{\prime}, x\right)$, and to every $x$ of $[a, b)$, a right-side interval $\left(x, x^{\prime \prime}\right)$, in each of which the oscillation of $f$ is less than $e / 4$. By the Heine-Borel covering theorem, there exists a finite set of points $x_{i}$ in $I$ such that the sum of the corresponding intervals $\left(x_{i}^{\prime}, x_{i}^{\prime \prime}\right) \equiv I_{i}$ (including the intervals $\left[a, a^{\prime \prime}\right)$ and $\left.\left(b^{\prime}, b\right]\right)$ covers $I$, and we may assume without loss of generality that the overlapping parts $\left(x_{i+1}^{\prime}, x_{i}^{\prime \prime}\right)$ of consecutive intervals $I_{i}$ (necessarily nonvacuous, as the $I_{i}$ are open) lie inside $\left(x_{i}, x_{i+1}\right)$. It can now be seen without difficulty that we can take as the required function $f_{e}$, a function defined as follows: It has the values $f\left(x_{i}\right)$, $f\left(x_{i}-0\right)$, and $f\left(x_{i}+0\right)$, respectively for $x=x_{i}$, for $x$ in $\left(x_{i-1}^{\prime \prime}, x_{i}\right)$, and for $x$ in $\left(x_{i}, x_{i+1}^{\prime}\right)$; and it is linear in the remaining intervals.

It is apparent how Theorem 2 can be derived by the use of the above three lemmas. We need only observe that the functions $f_{m}$ of Lemma 3 (with $f$ of Theorem 2) satisfy the relations (5) and (6) (with $f=f_{m}$ ) because they are in $V$, and have no common singularity

${ }^{10}$ The parenthesis will represent the open, and the bracket, the closed end of an interval. 
points with the functions $g_{n}, g$ (by their definition, in view of (5) and (6)). It is in fact known that two functions of bounded variation which have no common discontinuity points, are integrable with respect to each other. ${ }^{11}$

Proof of Theorem 3. Conditions (2) and (8) are equivalent [7] to (2) and the relation

$$
V_{a}^{x}\left(g_{n}\right) \rightarrow V_{a}^{x}(g), \quad n \rightarrow \infty ; x \text { in } I .
$$

Hence, denoting by $g_{n}=p_{n}-q_{n}, g=p-q$ the Jordan decompositions of the functions $g_{n}, g$, we readily see that all the conditions of Theorem B apply to the two sequences $\left\{p_{n}\right\},\left\{q_{n}\right\}\left(\int f d p_{n}, \int f d q_{n}\right.$ exist because $\int f d g_{n}$ exist [1]), and that therefore: $\int f d p_{n} \rightarrow \int f d p, \int f d q_{n} \rightarrow \int f d q$, and hence (3) is satisfied.

The theorem can also be proved directly as follows: ${ }^{12}$ Let

$$
s_{n k}=\sum_{i=1}^{r_{k}} f\left(u_{i, k}\right)\left[g_{n}\left(x_{i, k}\right)-g_{n}\left(x_{i-1, k}\right)\right]
$$

represent the $k$ th Riemann sum of a sequence of such sums which converges to $\int f d g_{n} \equiv J_{n}$ when $k \rightarrow \infty$, then:

$$
\left|J_{n}-s_{n k}\right| \leqq \sum_{i=1}^{r_{k}} \operatorname{Osc}_{i} f(x) V_{i}\left(g_{n}\right),
$$

the subscript $i$ referring to the $i$ th subinterval $\left(x_{i-1, k}, x_{i, k}\right)$. By (6), given a positive number $h$, there exists a number $K=K(h)$ such that $O_{k} \equiv \sum_{i} \operatorname{Osc}_{i} f(x) V_{i}(g)<h, k \geqq K(h)$. Now let $e$ be a preassigned positive number. Fixing first $k^{\prime} \geqq K(e / 2)$, we can then choose, in view of $\left(8^{\prime}\right)$, a number $N=N\left(e, k^{\prime}\right)$ so that for all $n \geqq N$, we have

$$
\left|V_{a}^{x_{i}, k^{\prime}}\left(g_{n}\right)-V_{a}^{x_{i}, k^{\prime}}(g)\right|<\frac{e}{4 r_{k^{\prime}} U} \equiv e^{\prime}, \quad U=\operatorname{Osc}_{I} f ; i=0,1, \cdots, r_{k^{\prime}} .
$$

Then

$$
\begin{array}{r}
\sum_{i=1}^{r_{k^{\prime}}} \operatorname{Osc}_{i} f(x) V_{i}\left(g_{n}\right)<O_{k^{\prime}}+2 e^{\prime} r_{k^{\prime}} U<e / 2+e / 2=e, \\
k^{\prime} \geqq K(e / 2)=k_{e} ; n \geqq N\left(e, k^{\prime}\right) .
\end{array}
$$

11 We note, incidentally, that by Lemma 3 and the first part of the proof of Lemma 2 , it follows that $a$ bounded function which has at most singularities of the first kind is integrable with respect to every function of $\boldsymbol{V}$ with which it has no common singularities.

12 This proof, which is different from that given in [3] for Theorem B, was found by the author in the winter of 1939 , when most of the results given in this paper were developed. 
Combining this with $\left(^{*}\right)$, and remembering that $e$ was arbitrarily assigned, we finally conclude that $\lim _{n \rightarrow \infty} J_{n}=\lim _{k} \lim _{n} s_{n k}=\int f d g$, that is, that we have (3).

Proof of Theorem 4. Let us suppose first that the limit function $v(x)$ occurring in (9) is unique. Then if $g_{n}=p_{n}-q_{n}$ represent the Jordan decompositions of the functions $g_{n}$, we find:

$2 p_{n}(x) \rightarrow v(x)+g(x)+g(a) \equiv p^{*}(x), 2 q_{n} \rightarrow v(x)-g(x)+g(a) \equiv q^{*}(x)$.

Since by (6) and (9), the integrals of $f$ with respect to $p^{*}$ and $q^{*}$ exist, we can now proceed exactly as in the first proof of Theorem 3 .

In the general case, let $\left\{J_{n^{\prime}}\right\}$ be an arbitrary convergent subsequence of $\left\{J_{n}\right\} \equiv\left\{\int f d g_{n}\right\}$. By Helly's theorem of choice, in view of (1) and (2), there exists a convergent subsequence $\left\{v_{n^{\prime \prime}}(x)\right\}$ of the sequence $\left\{v_{n^{\prime}}(x)\right\} \equiv\left\{V_{a}^{x}\left(g_{n^{\prime}}\right)\right\}$, and it follows by the preceding discussion that $J_{n^{\prime \prime}} \rightarrow \int f d g$. By a familiar reasoning it then follows that we also have the relation $J_{n} \rightarrow \int f d g$, that is, (3).

\section{BIBLIOGRAPHY}

1. E. W. Hobson, The Theory of Functions of a Real Variable, vol. 1, 3rd edition, 1927.

2. E. Helly, Über lineare Funktionaloperationen, Sitzungsberichte der Mathematisch-Naturwissenschaftliche Klasse der Akademie der Wissenschaften, Vienna, vol. 121 (1912), pp. 265-297.

3. J. Shohat, On mechanical quadratures, in particular, with positive coefficients, Transactions of this Society, vol. 42 (1937), pp. 461-496; p. 478.

4. H. E. Bray, Elementary properties of the Stieltjes integral, Annals of Mathematics, (2), vol. 20 (1918-1919).

5. T. H. Hildebrandt, Convergence of sequences of linear operations, this Bulletin, vol. 28 (1922), pp. 53-58.

6. O. Perron, Die Lehre von den Kettenbrïchen, 2 d edition, 1929, chap. 9.

7. C. R. Adams and J. A. Clarkson, On convergence in variation, this Bulletin, vol. 40 (1934), pp. 413-417; p. 414.

\section{Montana State College}

